\title{
EZH2 alteration driven by microRNA-524-5p and microRNA-324- $5 p$ promotes cell proliferation and temozolomide resistance in glioma
}

\author{
Tongle Zhi ${ }^{1,{ }^{*}}$, Tianfu $\mathrm{Yu}^{1{ }^{1,},}$, Minhong Pan ${ }^{2,{ }^{*}}$, Er Nie ${ }^{1}$, Weining $\mathbf{W u}^{1}$, Xiefeng Wang ${ }^{1}$, \\ Ning Liu ${ }^{1}$, Yongping You ${ }^{1}$, Yingyi Wang ${ }^{1}$ and Junxia Zhang ${ }^{1}$ \\ ${ }^{1}$ Department of Neurosurgery, The First Affiliated Hospital of Nanjing Medical University, Nanjing, China \\ ${ }^{2}$ Department of Pathology, The First Affiliated Hospital of Nanjing Medical University, Nanjing, China \\ *These authors have contributed equally to this work \\ Correspondence to: Yingyi Wang, email: lindsay_wang3431@126.com \\ Junxia Zhang, email: zjx232@njmu.edu.cn
}

Keywords: EZH2; miRNA; prognosis; TMZ; glioma

Received: June 28, $2017 \quad$ Accepted: August 27, $2017 \quad$ Published: October 24, 2017

Copyright: Zhi et al. This is an open-access article distributed under the terms of the Creative Commons Attribution License 3.0 (CC BY 3.0), which permits unrestricted use, distribution, and reproduction in any medium, provided the original author and source are credited.

\section{ABSTRACT}

Recent data have been shown that EZH2 is a critical oncogene via the repression of tumor suppressor genes in human cancers. In our study, we performed a genomewide miRNA screen with a bioinformatics analysis to identify EZH2 specific miRNAs. Of these miRNAs, miR-524-5p and miR-324-5p were decreased in glioma tissues, and confered poor prognosis for glioma patients. Upregulation of miR-524-5p and miR-324-5p reduced glioma cell proliferation and increased temozolomide (TMZ) chemosensitivity by targeting EZH2. Importantly, the effection of miR-524-5p and miR-324-5p on cell proliferation and TMZ chemosensitivity in glioma were reversed by expression of EZH2 cDNA. Further, miR-524-5p and miR-324-5p overexpression suppressed glioma growth and prolonged survival in an intracranial xenograft model. Multivariate Cox regression analysis revealed that miR-524-5p was an independent prognostic factor in gliobalstoma patients. Taken together, these data indicate that miRNA-driven EZH2 repression may provide evidence of the molecular mechanism for gliomagenesis and the novel therapeutic targets for glioma.

\section{INTRODUCTION}

The methyltransferase enhancer of zeste homolog 2 (EZH2), is the core catalytic element of polycomb repressive complex 2 (PRC2), and plays an important role in the regulation of cancer initiation, progression, invasion, and drug resistance. EZH2 catalyzes histone $\mathrm{H} 3$ lysine 27 methylation to form $\mathrm{H} 3 \mathrm{~K} 27 \mathrm{me}$, and act as an oncogene via the repression of tumor suppressor genes in human cancers [1-3]. EZH2 overexpression has been shown in colorectal cancers [4], esophageal cancers [5], bladder cancers [6], and lung cancers [7]. Associations have also been reported between EZH2 overexpression and poor prognosis in esophageal cancers [8], breast cancers [9], renal cell carcinomas [10] and childhood intracranial ependymoma [11]. Our recent data have shown that increased EZH2 expression was associated with glioma grade, and high expression of EZH2 in GBM (glioblastoma) was determined to be a strong and independent predictor of short overall survival [12].

MicroRNAs constitute a class of small non-coding RNA molecules that function as post-transcriptional gene regulators and have been increasingly recognized as oncogenes or tumor suppressors [13, 14]. In human colon cancer, miR-506 inhibits cell proliferation and metastasis by binding the 3'UTR of EZH2 [15]. And in liver cancer, miR-101 represses tumor progression and sensitizes cancer cells to chemotherapeutic treatment through directly targeting EZH2 [16]. Also miR-101 is 
down-regulated in GBM cells, resulting in increased EZH2 expression and enhanced GBM cell proliferation, migration, and angiogenesis [17]. However, few studies of systematic mining EZH2 specific miRNA signature in human cancers have been reported. Thus, in this manuscript, we identified EZH2 specific miRNAs using integrated analysis of miRNA and mRNA arrays. Of these miRNAs, miR-524-5p and miR-324-5p confered poor prognosis for glioma patients and inhibited glioma cell proliferation by targeting EZH2.

\section{RESULTS}

\section{MiRNA expression profiling for EZH2 specific miRNA signature}

To identify the signature of EZH2 associated miRNA, miRNA and mRNA expression profiling of 64 GBM specimens from CGGA was employed. Pearson correlation was performed to analyze the relationships of EZH2 and all miRNA values using matlab software. 57 positively and 71 negatively correlated miRNAs with EZH2 expression were detected. Then heat map of these miRNAs was shown in profiling data with 158 gliomas (Figure 1A). Only the miRNAs with negatively significant correlation with EZH2 level were considered as EZH2 specific miRNA signature.

We analyzed the overall survival of GBM patients with EZH2 specific miRNA signature using matlab software, and found 7 miRNAs overexpression confer a better prognosis in GBM patients, as shown in Figure 1B. Further we used miRNA target analysis to explore whether EZH2 was a direct target of these miRNAs. Among them, two miRNAs (miR-524-5p and miR-324-5p), were predicted to bind to EZH2 3'UTR. The samples expressing lower level of miR-524-5p were associated with decreased survival relative to those with higher level in all gliomas $(\mathrm{P}<0.0001)$ and high grade gliomas (grade III and grade IV) $(\mathrm{P}=0.0020)$ (Figure 1C). And similar results of miR$324-5 p$ were detected in all gliomas $(\mathrm{P}<0.0001)$ and high grade glioma $(\mathrm{P}=0.0430)$ (Figure $1 \mathrm{C})$. These data indicate that miR-524-5p and miR-324-5p overexpression correlates with a significantly better survival outcome.

Further we check miR-524-5p and miR-324-5p expression in 158 glioma tissues. The level of miR-524$5 \mathrm{p}$ was significant lower in high grade gliomas than in low grade gliomas $(\mathrm{P}<0.0001)$ (Figure 1D). Moreover, GBM demonstrated a significant decrease in miR-524$5 \mathrm{p}$ expression compared with that observed in low grade gliomas $(\mathrm{P}<0.0001)$ and anaplastic gliomas (grade III) (P $=0.0227$ ). And miR-324-5p was also inversely associated with tumor grade $(\mathrm{P}<0.0001)$. Also, we checked 8 normal brain tissues, 8 grade II glioma tissues and 8 grade IV glioma tissues, and found the similar data shown in Figure
1E. Over all, these findings suggest that EZH2 specific miRNAs miR-524-5p and miR-324-5p may play an important role in glioma development.

\section{Critical role of miR-524-5p and miR-324-5p in glioma cell proliferation}

To further explore the role of miR-524-5p and miR-324-5p in cell proliferation, we performed MTT and colony formation assays. Overexpression of miR-524-5p significantly inhibited glioma cell proliferation in U87 and U251 cells by MTT assay (Figure 2A). And cells exhibited a significant reduction in colony formation after 2 weeks of miR-524-5p treatment compared with the control group (Figure 2B). Also MTT and colony formation assays showed that miR-324-5p overexpression suppresses cell proliferation in both U87 and U251 glioma cells (Figure $2 \mathrm{~A}$ and $2 \mathrm{~B})$.

\section{EZH2 is a direct target of miR-524-5p and miR- 324-5p}

To determine the association of miR-524-5p and miR-324-5p with EZH2, Western blot and reporter assays were used. Western blot analysis showed that EZH2 expression was down-regulated in glioma cells over-expressing miR-524-5p or miR-324-5p (Figure $3 A)$. In addition, we created pGL3-WT-EZH2-3'UTR and pGL3-MUT-EZH2-3'UTR plasmids for miR-524-5p or miR-324-5p, respectively. Reporter assays revealed that induction of miR-524-5p or miR-324-5p triggered a marked decrease of luciferase activity from pGL3-WTEZH2-3'UTR but produced no change in the luciferase activity from pGL3-MUT-EZH2-3'UTR (Figure 3B). These data indicate that miR-524-5p or miR-324-5p directly modulated EZH2 expression by binding to the respective $3^{\prime} \mathrm{UTR}$.

\section{Expression of EZH2 overrides miR-524-5p and miR-324-5p function}

Having demonstrated the profound effects of miR524-5p and miR-324-5p on tumor suppression, we sought to examine the importance of EZH2 in miR-524-5p and miR-324-5p mediated cell proliferation. We transfected miR-524-5p mimics or miR-324-5p mimics together with and without EZH2 plasmid lacking 3'UTR into glioma cells (Figure 4A). Overexpression of EZH2 significantly rescued cell proliferation in U87 and U251 cells (Figure 4B and 4C). Similarly, expression of EZH2 largely abrogated miR-324-5p effects on cell proliferation (Figure 4B and 4C). These suggest that EZH2 is a critical target of miR-524-5p and miR-324-5p involved in glioma cell proliferation. 


\section{MiR-524-5p and miR-324-5p increase TMZ chemosensitivity in glioma}

Recent data have shown that inhibition of EZH2 promotes chemotherapeutic drug temozolomide (TMZ) chemosensitivity in glioblastoma cells [18]. Thus, we explore whether miR-524-5p and miR-324-5p play key roles in TMZ resistance. The MTT assay showed that upregulation of miR-524-5p significantly reduced the viability of U87 and U251 cells after TMZ treatment (Figure 5A). And EZH2 overexpression could reverse miR-524-5p role in TMZ chemosensitivity. Also, the same results of miR-324-5p in TMZ chemosensitivity were observed (Figure 5A). Further, we evaluated the expression levels of DKK1 and p21, which were targets of EZH2 $[19,20]$ and associated with chemosensitivity of alkylating drugs $[21,22]$. EZH2 reduction elevated DKK1 and p21 were elevated when cells were treated by si-EZH2, miR-524-5p and miR-324-5p mimics, as shown in Figure 5B. Glioma stem-like cells has been identified as a main object for TMZ resistance. Thus, we performed clonogenic assay to detect the effect of TMZ on glioma stem-like cells (U87-GS cells). Compared to the control group, the percentage of positive wells (positive well means 1 cell colony/well) was lower, and the spheres were smaller in miR-524-5p or miR-324-5p treated U87-GS cells under TMZ treatment (Figure 5C). EZH2 overexpression could eliminate miR-324-5p or miR-324$5 \mathrm{p}$ effects on sphere formation under TMZ treatment. These suggested that miR-524-5p and miR-324-5p may modulate TMZ resistance in glioma cells.

\section{MiR-524-5p and miR-324-5p suppress tumor growth in an intracranial xenograft model}

To further evaluate the effects of miR-524-5p and miR$324-5 \mathrm{p}$ on tumor growth in vivo, we established intracranial xenograft tumors in nude mice. U87 cells were pretreated with a lentivirus containing a luciferase reporter. As shown in Figure 6A, when miR-524-5p was overexpressed, the
A

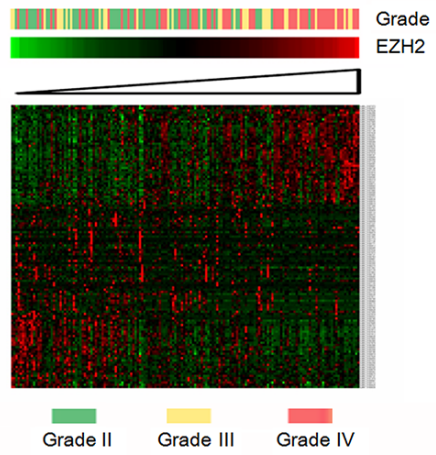

B

\begin{tabular}{llllll}
\hline MiRNA & \multicolumn{2}{l}{ Survival } & & \multicolumn{2}{l}{$E Z H 2$ association } \\
\cline { 2 - 3 } \cline { 6 - 7 } & $H R$ & $P$ & & $R$ & $P$ \\
\hline hsa-miR-328 & 0.9997 & 0.0002 & & -0.3072 & 0.0135 \\
hsa-miR-524-5p & 0.9845 & 0.0004 & & -0.2481 & 0.0480 \\
hsa-miR-598 & 0.9998 & 0.0117 & & -0.2541 & 0.0428 \\
hsa-miR-29b-2* & 0.9994 & 0.0231 & & -0.4056 & 0.0008 \\
hsa-miR-935 & 0.9998 & 0.0250 & & -0.2821 & 0.0239 \\
hsa-miR-628-5p & 0.9998 & 0.0305 & & -0.4579 & 0.0001 \\
hsa-miR-324-5p & 0.9998 & 0.0328 & & -0.2860 & 0.0220 \\
\hline
\end{tabular}

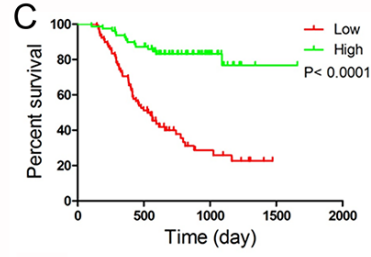

miR-524-5p, all glioma

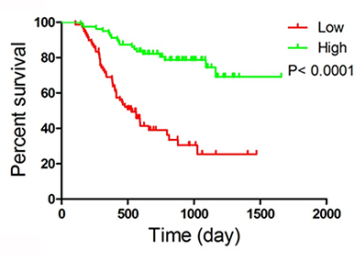

miR-324-5p, all glioma

\section{D}

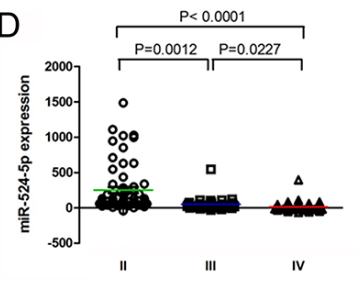

E

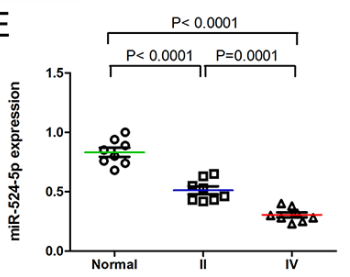

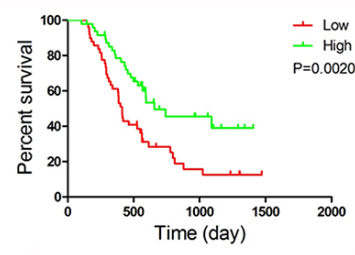

miR-524-5p, high grade glioma

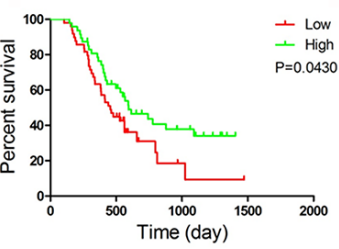

miR-324-5p, high grade glioma
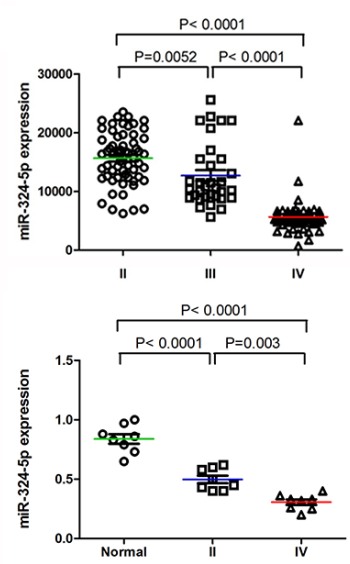

Figure 1: MiR-524-5p and miR-324-5p were identified as key EZH2 specific miRNAs. (A) Heatmap of EZH2 and EZH2 associated miRNAs in glioma patients sorted by level of EZH2 expression in CGGA data. (B) Seven EZH2 specific miRNAs confer a better prognosis in GBM patients. (C) Kaplan-Meier survival curves for miR-524-5p and miR-324-5p in all gliomas and high grade gliomas. Patients with low levels of miR-524-5p or miR-324-5p had a significantly worse outcome. (D) Levels of miR-524-5p and miR-324-5p were analyzed in different glioma tissues in CGGA data. (E) Levels of miR-524-5p and miR-324-5p were analyzed in normal brain tissues and glioma tissues by PCR. 

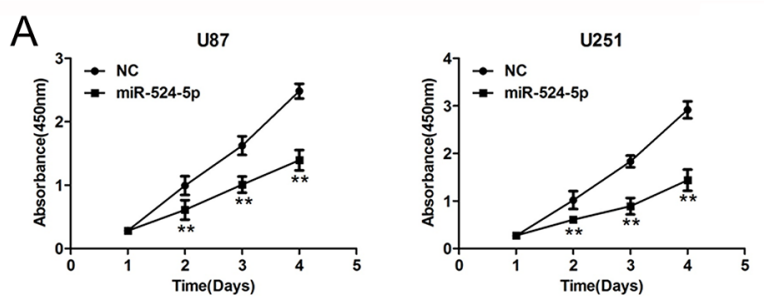

U87

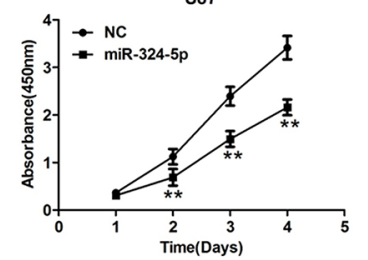

U251
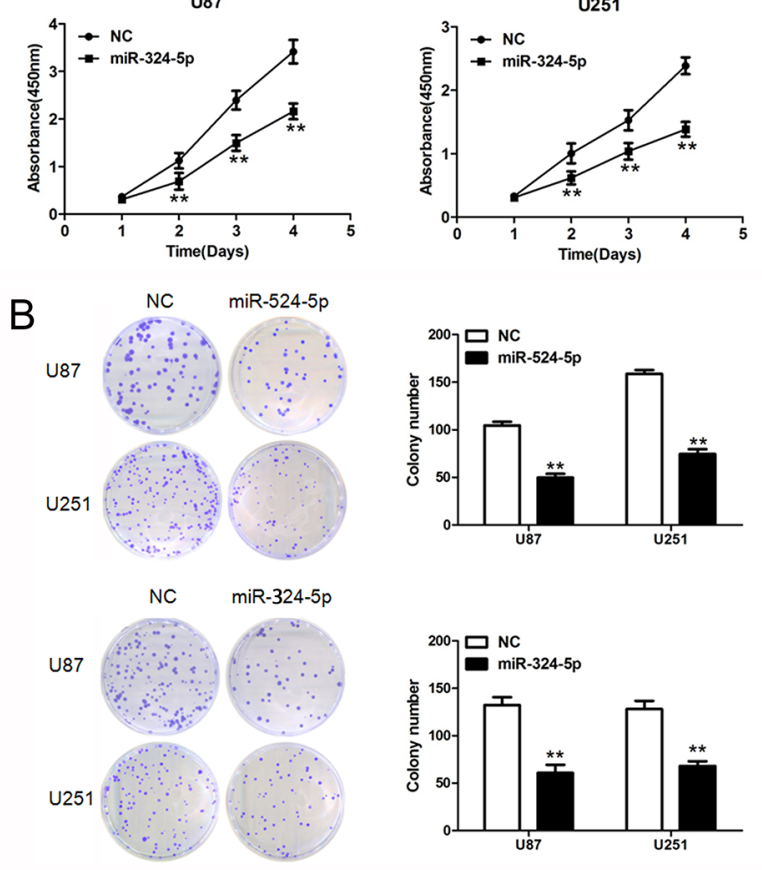

Figure 2: Overexpression of miR-524-5p or miR-324-5p inhibits tumor growth in vitro. (A) Cells were treated with miR524-5p or miR-324-5p mimics, and subjected to MTT assay. Data are presented as the means of triplicate experiments. (B) Cells were treated with miR-524-5p or miR-324-5p mimics, and subjected to colony formation assay. Data are presented as the means of triplicate experiments, ${ }^{* *} \mathrm{P}<0.01$.
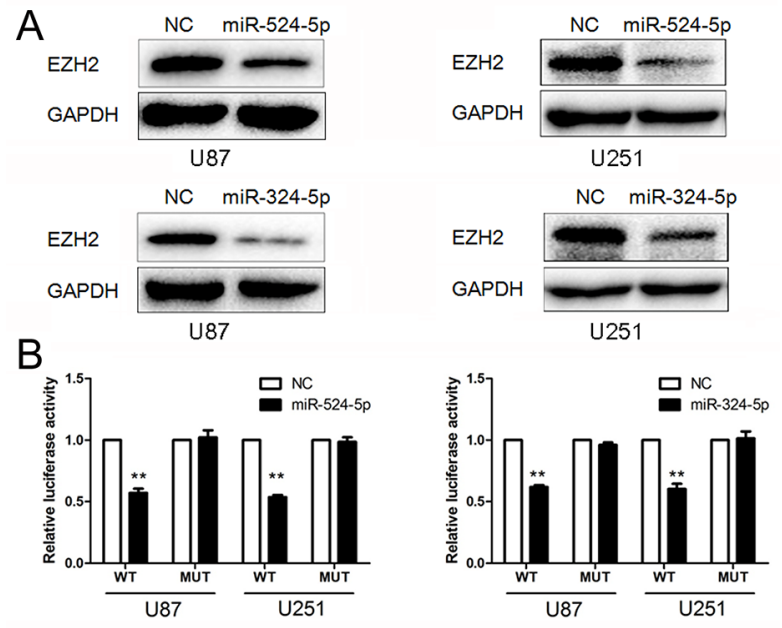

Figure 3: MiR-524-5p and miR-324-5p target EZH2. (A) Western blot analysis of lysates from cells transfected by miR-524-5p or miR-324-5p mimics probed with EZH2 antibody. GAPDH was served as the loading control. (B) pGL3-WT-EZH2-3'UTR-Luc and pGL3MUT-EZH2-3'UTR-Luc reporters were transfected into glioma cells treated by miR-524-5p or miR-324-5p mimics. Luciferase activity was determined $48 \mathrm{~h}$ after transfection. The ratio of normalized sensor to control luciferase activity was shown. Error bars represent standard deviation and were obtained from three independent experiments, ${ }^{* *} \mathrm{P}<0.01$. 
intracranial tumor significantly decreased compared with the corresponding control group $(\mathrm{P}<0.01)$. Compared with the control group, miR-524-5p-treated group showed prolonged survival until the end of the observation (on day 35) (Figure 6A). The similar results of miR-324-5p were observed (Figure 6B). Furthermore, immunohistochemistry
A

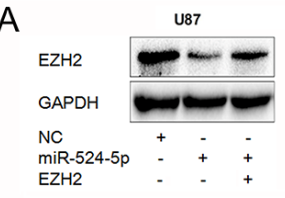

$\mathrm{B}$

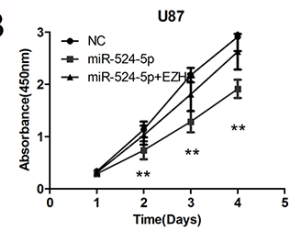

C NC miR-524-5p miR-524-5p+EZH2

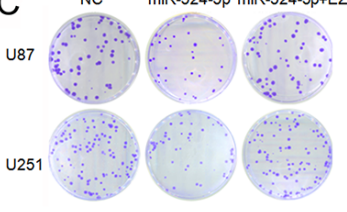

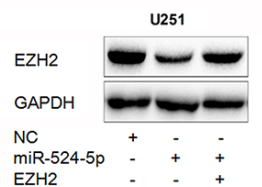

U251
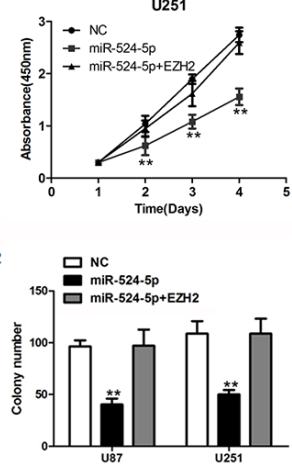

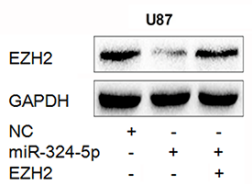

U87
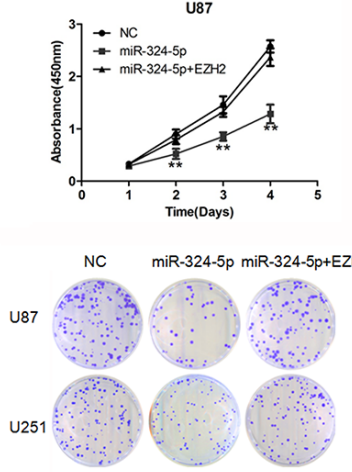

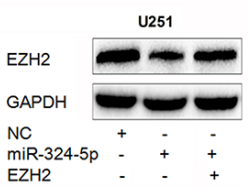

U251
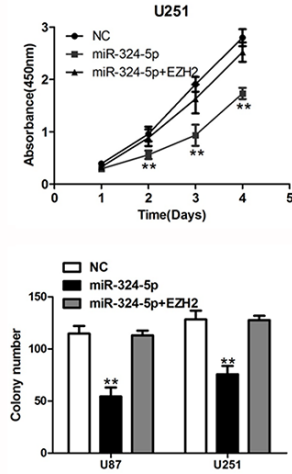

Figure 4: EZH2 is crucial for miR-524-5p and miR-324-5p signaling in glioma. (A) Western blot analysis of lysates from cells transfected by miR-524-5p or miR-324-5p mimics alone, or in combination with EZH2 cDNA probed with EZH2 antibody. GAPDH was served as the loading control. (B and C) MTT and colony formation assays were measured in U87 and U251 glioma cell lines after the cells were treated with miR-524-5p or miR-324-5p mimics alone, or in combination with EZH2 cDNA. Results are representative of at least three independent experiments, ${ }^{* *} \mathrm{P}<0.01$.

A
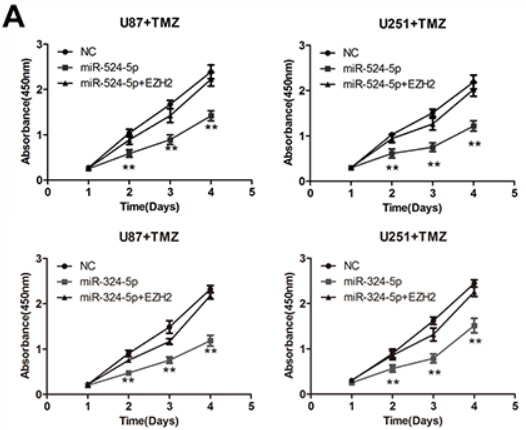

C

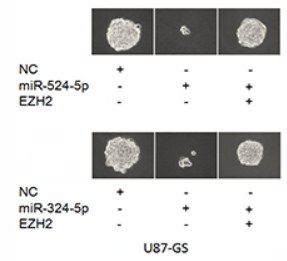

B
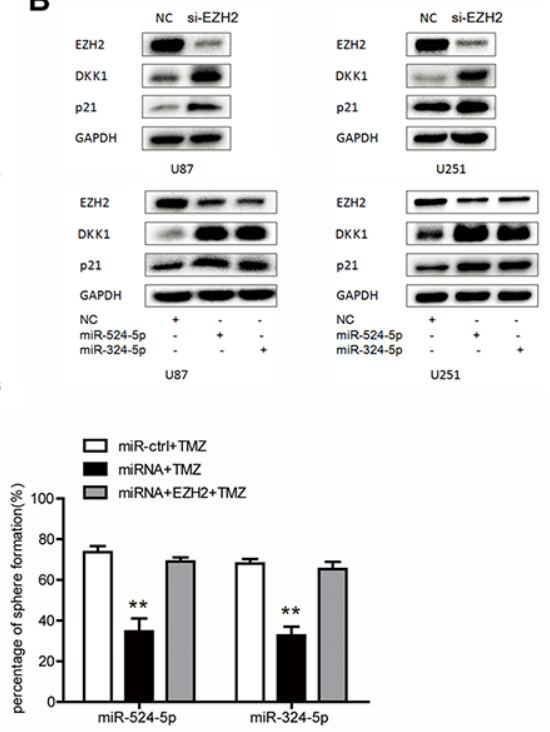

Figure 5: MiR-524-5p and miR-324-5p increases TMZ chemosensitivity in glioma. (A) MTT assay was measured in U87 and U251 glioma cell lines by TMZ treatment after the cells were transfected with miR-524-5p or miR-324-5p mimics alone, or in combination with EZH2 cDNA. (B) Western blot analysis of lysates from cells transfected by si-EZH2, miR-524-5p or miR-324-5p mimics probed with EZH2, DKK1 and p21 antibody. GAPDH was served as the loading control. (C) Clonogenic assay was used to measure the sphere formation of glioma stem-like cells treated TMZ for $15 \mathrm{~d}$. Representative photomicrographs of new clonal sphere (left) and statistical analysis of the percentage of positive wells (right). Results are representative of at least three independent experiments, ${ }^{* *} \mathrm{P}<0.01$. 
assay showed the decreased expression of EZH2 and increased expression of $\mathrm{p} 21$ and DKK 1 after overexpression of miR-324-5p or miR-524-5p, which were consistent with the in vitro results (Supplementary Figure 1). Taken together, these findings demonstrate that both miR-524-5p and miR$324-5 p$ inhibited glioma malignant progression in vivo.

\section{MiR-524-5p is an independent prognostic factor in GBM patients}

As shown in Supplementary Table 1, high expression of miR-524-5p was significantly associated with IDH1 mutation, PCNA expression, but not associated with MGMT promoter methylation. Then we performed univariate cox regression analysis using clinical and genetic variables for $64 \mathrm{GBM}$ patients. We found that high expression of miR-524-5p, high KPS score and total resection were statistically associated with overall survival, while IDH1 mutation and MGMT promoter methylation were not associated with overall survival (Table 1). Multivariate Cox regression analysis revealed that miR-524-5p expression, $\mathrm{Ki}-67$ expression and total resection were correlated independently with overall survival when considering gender, KPS score, IDH1 mutation, EGFR expression and PCNA expression $(\mathrm{P}<$ 0.3 , univariate cox regression analysis).

\section{MiR-524-5p could be used to sub-classify gliomas in combination with EZH2 expression}

To further detail the role of miR-524-5p in glioma survival, we designed a glioma classification model based on miR-524-5p and EZH2 expression level. As shown in Figure $6 \mathrm{C}, \mathrm{GBM}$ patients with low EZH2 and high miR524-5p had the longest OS, whereas GBM patients with high EZH2 and low miR-524-5p had the shortest (median $\mathrm{OS}=529.6$ vs. 349.8 days; logrank test, $\mathrm{P}=0.0099$ ). And the similar results in high grade gliomas and all gliomas were observed. High EZH2 and low miR-524-5p in glioma patients was associated with the longest OS (median OS $=460.6$ days; logrank test, $\mathrm{P}<0.0001$ ). Therefore, we conclude that classification combining miR-524-5p and EZH2 expression represents a more precise biological property and prognosis.
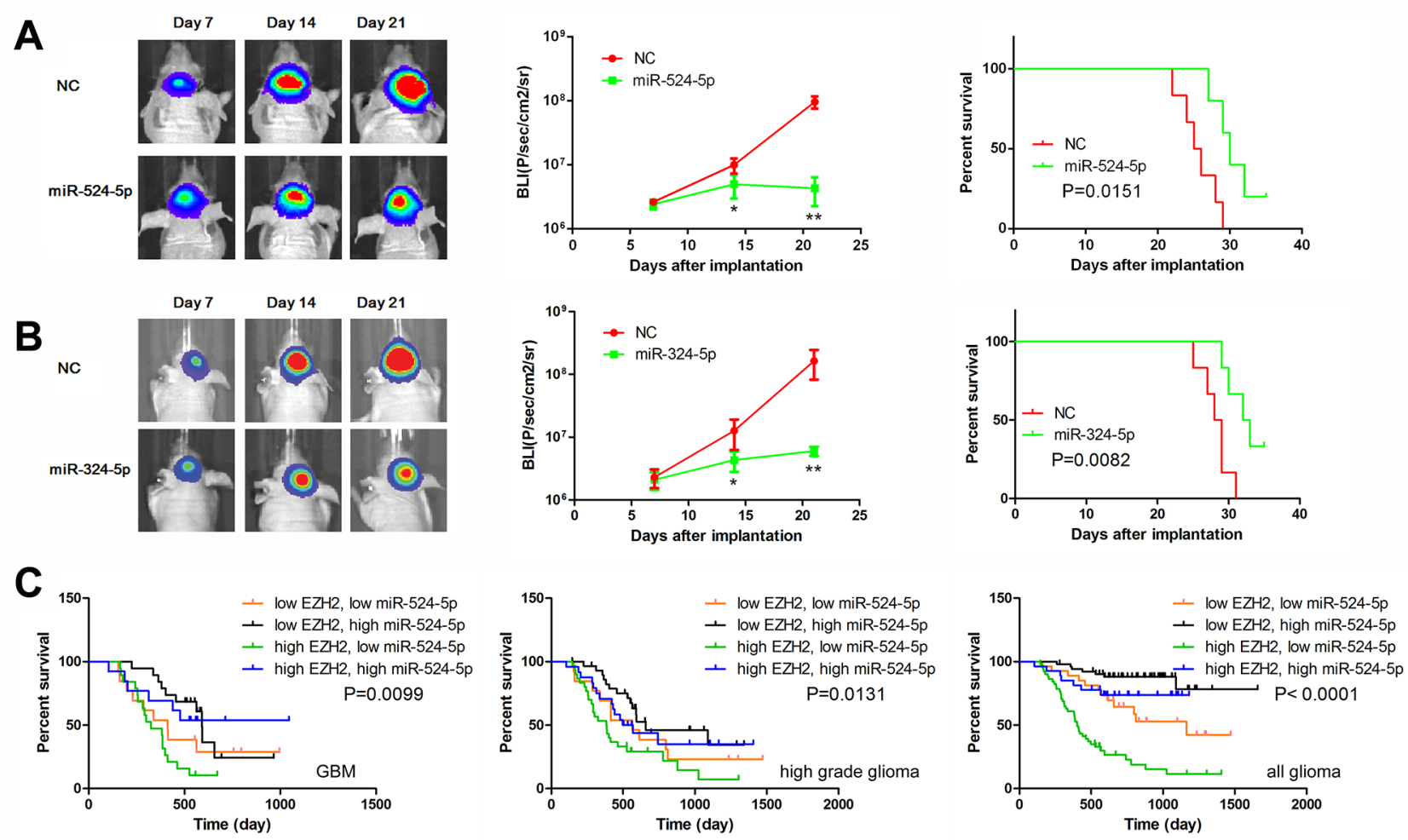

Figure 6: MiR-524-5p suppresses tumor growth in an intracranial xenograft model. (A, B) U87 cells pretreated with lentivirus containing a luciferase reporter were implanted into the brains of nude mice, and tumor formation was assessed by bioluminescence imaging. Changes in bioluminescent signal were detected at days 7, 14, and 21 after implantation. Overall survival of nude mice was determined by Kaplan-Meier survival curves and log-rank test was used to assess the statistical significance of the differences. ${ }^{*} \mathrm{P}<0.05,{ }^{* *} \mathrm{P}<0.01$. (C) Combining analysis of miR-524-5p and EZH2 expression by KaplanMeier survival curves in different glioma patients of CGGA data. 
Table 1: Cox regression analyses of miR-524-5p expression and pathologic characteristics in relation to overall survival in GBM

\begin{tabular}{|c|c|c|c|c|}
\hline \multirow[t]{2}{*}{ Variable } & \multicolumn{2}{|c|}{ Univariable regression } & \multicolumn{2}{|c|}{ Multivariable regression } \\
\hline & HR & $\mathbf{P}$ & HR & $\mathbf{P}$ \\
\hline Gender (female/ male) & 1.440 & 0.276 & 1.542 & 0.287 \\
\hline Increasing age & 1.008 & 0.541 & & \\
\hline KPS score $(>80)$ & 0.363 & 0.001 & 0.496 & 0.089 \\
\hline Total resection & 0.550 & 0.022 & 0.360 & 0.031 \\
\hline IDH1 mutation & 0.494 & 0.142 & 0.667 & 0.479 \\
\hline MGMT promoter methylation & 1.208 & 0.645 & & \\
\hline High miR-524-5p & 0.403 & 0.005 & 0.336 & 0.012 \\
\hline High Ki-67 & 1.059 & 0.050 & 2.529 & 0.027 \\
\hline High EGFR & 1.483 & 0.223 & 0.871 & 0.732 \\
\hline High MGMT & 1.077 & 0.823 & & \\
\hline High MMP9 & 0.744 & 0.421 & & \\
\hline High P53 & 1.165 & 0.688 & & \\
\hline High PCNA & 1.785 & 0.069 & 1.805 & 0.131 \\
\hline
\end{tabular}

\section{DISCUSSION}

Here, we present the first study to integrate a genome-wide miRNA screen with a bioinformatics analysis derived from clinical specimens to identify clinically pertinent EZH2 specific miRNAs. To our surprise, 71 miRNAs are negatively significant correlated with EZH2 level and 7 EZH2 specific miRNAs confer a better prognosis in GBM patients. We characterize two candidates, miR-524-5p and miR-324-5p, which bind to EZH2 3'UTR to down-regulate EZH2 expression, thereby suppressing glioma cell proliferation. Importantly, restoring EZH2 expression could override miR-524-5p and miR-324-5p function in glioma cells. These data indicate that miRNA-driven EZH2 repression may underlie the molecular mechanism for gliomagenesis and represent a novel therapeutic target for glioma.

Accumulating data have been validated that miR$524-5 \mathrm{p}$ is a key tumor suppressors in several human cancers. In melanoma cells, miR-524-5p directly interacts with the 3'-untranslated regions of both BRAF and ERK2 to inhibit MAPK/ERK signaling, cell proliferation, and cell migration [23]. Another study has shown that miR$524-5 p$ behaves as a tumor suppressor by negatively targeting Jagged-1 and Hes-1 in gliomas [24]. Here, we found miR-524-5p is down-regulated in glioma tissues and associated with tumor grade. Low expression of miR-524$5 \mathrm{p}$ in GBM is determined to be a strong and independent predictor of short overall survival. Overexpression of miR524-5p inhibits glioma cell growth in vitro and in vivo.
Further a combined index of miR-524-5p and EZH2 expressions better reflects prognosis of glioma patients.

$\mathrm{EZH} 2$, as the core element of PRC2, mediates histone methylation and recruits DNA methyltransferase in the silencing of a variety of genes, including miRNAs $[25,26]$. In gastric cancer and glioma cells, knockdown of EZH2 not only impacted H3K27 trimethylation but also reduced DNMT1 presence on the miR-200b/a/429 promoter [27]. In human nasopharyngeal carcinoma, EZH2 inhibits miR1 transcription via promoter binding activity, leading to enhanced expression of Endothelin-1 (ET-1) which is suppressed by miR-1 targeting of ET-1 3'UTR [28]. In aggressive B-Cell lymphomas, Myc, HDAC3, and PRC2 are tethered to the miR-29 promoter regions as a co-repressor complex to down-regulate miR29 expression through histone deacetylation and trimethylation [29]. In our results, miR-1, miR29b, miR-29c are listed in EZH2 specific miRNAs. Thus, the detailed mechanism involved in EZH2 specific miRNAs in glioma progression needs further investigation.

In summary, we identify EZH2 specific miRNAs through a genome-wide miRNA screen. MiR-524-5p and miR-324-5p exhibit a strong tumor-suppressive effect by targeting EZH2. Further, miR-524-5p was an independent prognostic factor in GBM patients. These data indicate that miRNA-driven EZH2 repression provides evidence of the molecular mechanism for gliomagenesis and the novel therapeutic targets for glioma. 


\section{MATERIALS AND METHODS}

\section{Patients and samples}

We performed miRNA and mRNA profiling upon 158 glioma samples collected from the Chinese Glioma Genome Atlas (CGGA, http://www.cgeg.org.cn/) including 61 gliomas of grade II, 33 gliomas of grade III, and 80 GBMs. 8 normal brain tissues, 8 grade II glioma tissues and 8 grade IV glioma tissues were obtained from The First Affiliated Hospital of Nanjing Medical University. This study was approved by the institutional review boards of all hospitals involved in the study, and written informed consent was obtained from all the patients who were selected.

\section{Cell culture and treatment}

Human glioma cells (U87 and U251) were obtained from the Chinese Academia Sinica cell repository (Shanghai, China). Cells were maintained in Dulbecco's modified Eagle's medium (DMEM, Gibco) supplemented with $10 \%$ fetal bovine serum, and incubated at $37^{\circ} \mathrm{C}$ with 5\% CO2. Glioma stem-like cells (U87-GS), single-cell populations which came from adherent U87 cells, were resuspended in DMEM/F12 (Gibco), supplemented with $\mathrm{N} 2(1 / 100$, Invitrogen), B27(1/50, Invitrogen), $10 \mathrm{ng} / \mathrm{ml}$ recombinant human basic fibroblast growth factor (FGF, Invitrogen) and $10 \mathrm{ng} / \mathrm{ml}$ recombinant human epidermal growth factor (EGF, Invitrogen). Oligonucleotides were chemically synthesized and purified by highperformance liquid chromatography (GenePharma, Shanghai, China). Then miRNA mimics were transfected using Lipofectamine 2000 (Invitrogen). Lentivirus overexpressing miR-524-5p and ontaining a luciferase reporter were obtained from genechem (Shanghai, China).

\section{Colony formation assay and clonogenic assay}

4000 glioma cells from each group were seeded into new dishes. The medium was replaced every other day for 8 days, and the cells were stained with crystal violet at the end of the time course prior to the capture of the representative images via camera. U87-GS spheres were dissociated with TrypLETM Express (Gibco) and resuspended in sphere medium. Disaggregated spheres after trasfection were seeded in 96-well plates at clonal density ( 1 cell per well) and cultured in sphere medium with TMZ treatment. To renew growth factors supply, fresh sphere medium was added every $5 \mathrm{~d}$. $15 \mathrm{~d}$ later, we counted the percentage of spheres formation. The assays were repeated at least 3 times.

\section{Western blot analysis}

Equal amounts of protein per lane were separated by $8 \%$ SDS-polyacrylamide gel and transferred to PVDF membrane. The membrane was blocked in 5\% skim milk for $1 \mathrm{~h}$ and then incubated with a specific antibody for $2 \mathrm{~h}$. The antibodies used in this study were: antibodies to EZH2, DKK1, p21 (Cell Signaling Technology, USA). The antibody against GAPDH (Santa Cruz, USA) was used as control. The specific protein was detected by using a SuperSignal protein detection kit (Pierce, USA). The band density of specific proteins was quantified after normalization with the density of GAPDH.

\section{Luciferase reporter assay}

The human $\beta$-catenin 3'UTR were amplified and cloned into the $\mathrm{XbaI}$ site of the pGL3-control vector (Promega, USA), downstream of the luciferase gene, to generate the plasmids pGL3-WT-EZH2-3'UTR. pGL3MUT-EZH2-3'UTR plasmids were generated from pGL3-WT-EZH2-3'UTR by deleting the binding site. For the luciferase reporter assay, cells were cultured in 96-well plates, transfected with the plasmids and miRNA mimics using Lipofectamine 2000. 48h after transfection, luciferase activity was measured using the Luciferase Assay System (Promega).

\section{Nude mouse glioma intracranial model}

U87 cells were transfected with lentivirus overexpressing miR-524-5p or miR-324-5p and ontaining a luciferase reporter in vitro for 2 days. A total of 5 $\times 10^{5}$ U87 cells infected with virus were implanted stereotactically to establish intracranial gliomas using cranial guide screws. Mice were imaged for Fluc activity using bioluminescence imaging (BLI) on day 7, 14 and 21 .

\section{Statistical analysis}

Descriptive statistics, including mean and $\pm \mathrm{SE}$, along with independent sample t-tests, and the Pearson correlation were used to determine significant differences. Kaplan-Meier analysis was employed to assess the survival rate of patients. $\mathrm{P}<0.05$ was considered a significant difference.

\section{Abbreviations}

GBM, Glioblastoma multiforme; miRNAs, MicroRNAs; CGGA, Chinese Glioma Genome Atlas; TMZ, Temozolomide; PRC2, Polycomb repressive complex 2; ET-1, Endothelin-1; BLI, Bioluminescence imaging.

\section{Author contributions}

TLZ and TFY contributed to the conception and design of the experiment and performed it. MHP, EN, WNW, XFW, NL, and YPY provided the technical support. YTF offered data analysis. TLZ wrote the manuscript, and 
then provided ideas, discussion and critically reviewed the manuscript.

\section{GRANT SUPPORT}

This work was supported by grants from the National Key Research and Development Plan (2016YFC0902500), National Natural Science Foundation of China (81672501, 81302185), Jiangsu Province's Natural Science Foundation (20151585), the Program for Advanced Talents within Six Industries of Jiangsu Province (2015-WSN-036, 2016-WSW-013), and the Priority Academic Program Development of Jiangsu Higher Education Institutions (PAPD).

\section{CONFLICTS OF INTEREST}

The authors declare that there are no conflicts of interest.

\section{REFERENCES}

1. Kim KH, Roberts CW. Targeting EZH2 in cancer. Nat Med. 2016; 22:128-134.

2. Jiang T, Wang Y, Zhou F, Gao G, Ren S, Zhou C. Prognostic value of high EZH2 expression in patients with different types of cancer: a systematic review with meta-analysis. Oncotarget. 2016; 7:4584-4597. https://doi.org/10.18632/ oncotarget.6612.

3. Kondo Y. Targeting histone methyltransferase EZH2 as cancer treatment. J Biochem. 2014; 156:249-257.

4. Kodach LL, Jacobs RJ, Heijmans J, van Noesel CJ, Langers AM, Verspaget HW, Hommes DW, Offerhaus GJ, van den Brink GR, Hardwick JC. The role of EZH2 and DNA methylation in the silencing of the tumour suppressor RUNX3 in colorectal cancer. Carcinogenesis. 2010; 31:1567-1575.

5. Koumangoye RB, Andl T, Taubenslag KJ, Zilberman ST, Taylor CJ, Loomans HA, Andl CD. SOX4 interacts with EZH2 and HDAC3 to suppress microRNA-31 in invasive esophageal cancer cells. Mol Cancer. 2015; 14:24.

6. Martinez-Fernandez M, Duenas M, Feber A, Segovia C, Garcia-Escudero R, Rubio C, Lopez-Calderon FF, DiazGarcia C, Villacampa F, Duarte J, Gomez-Rodriguez MJ, Castellano D, Rodriguez-Peralto JL, et al. A Polycombmir200 loop regulates clinical outcome in bladder cancer. Oncotarget. 2015; 6:42258-42275. https://doi.org/10.18632/ oncotarget.5546.

7. Geng J, Li X, Zhou Z, Wu CL, Dai M, Bai X. EZH2 promotes tumor progression via regulating VEGF-A/AKT signaling in non-small cell lung cancer. Cancer Lett. 2015; 359:275-287.

8. Liu F, Gu L, Cao Y, Fan X, Zhang F, Sang M. Aberrant overexpression of EZH2 and H3K27me3 serves as poor prognostic biomarker for esophageal squamous cell carcinoma patients. Biomarkers. 2016; 21:80-90.

9. Wang X, Hu B, Shen H, Zhou H, Xue X, Chen Y, Chen S, Han Y, Yuan B, Zhao H, Zhi Q, Kuang Y. Clinical and prognostic relevance of EZH2 in breast cancer: a metaanalysis. Biomed Pharmacother. 2015; 75:218-225.

10. Wang Y, Chen Y, Geng H, Qi C, Liu Y, Yue D. Overexpression of $\mathrm{YB} 1$ and EZH2 are associated with cancer metastasis and poor prognosis in renal cell carcinomas. Tumour Biol. 2015; 36:7159-7166.

11. Li AM, Dunham C, Tabori U, Carret AS, McNeely PD, Johnston D, Lafay-Cousin L, Wilson B, Eisenstat DD, Jabado N, Zelcer S, Silva M, Scheinemann K, et al. EZH2 expression is a prognostic factor in childhood intracranial ependymoma: a Canadian Pediatric Brain Tumor Consortium study. Cancer. 2015; 121:1499-1507.

12. Zhang J, Chen L, Han L, Shi Z, Pu P, Kang C. EZH2 is a negative prognostic factor and exhibits pro-oncogenic activity in glioblastoma. Cancer Lett. 2015; 356:929-936.

13. Shi L, Cheng Z, Zhang J, Li R, Zhao P, Fu Z, You Y. hsamir-181a and hsa-mir-181b function as tumor suppressors in human glioma cells. Brain Res. 2008; 1236:185-193.

14. Shi Z, Zhang J, Qian X, Han L, Zhang K, Chen L, Liu J, Ren Y, Yang M, Zhang A, Pu P, Kang C. AC1MMYR2, an inhibitor of dicer-mediated biogenesis of Oncomir miR-21, reverses epithelial-mesenchymal transition and suppresses tumor growth and progression. Cancer Res. 2013; 73:5519-5531.

15. Zhang Y, Lin C, Liao G, Liu S, Ding J, Tang F, Wang Z, Liang X, Li B, Wei Y, Huang Q, Li X, Tang B. MicroRNA-506 suppresses tumor proliferation and metastasis in colon cancer by directly targeting the oncogene EZH2. Oncotarget. 2015; 6:32586-32601. https:// doi.org/10.18632/oncotarget.5309.

16. Xu L, Beckebaum S, Iacob S, Wu G, Kaiser GM, Radtke A, Liu C, Kabar I, Schmidt HH, Zhang X, Lu M, Cicinnati VR. MicroRNA-101 inhibits human hepatocellular carcinoma progression through EZH2 downregulation and increased cytostatic drug sensitivity. J Hepatol. 2014; 60:590-598.

17. Smits M, Nilsson J, Mir SE, van der Stoop PM, Hulleman E, Niers JM, de Witt Hamer PC, Marquez VE, Cloos J, Krichevsky AM, Noske DP, Tannous BA, Wurdinger T. miR-101 is down-regulated in glioblastoma resulting in EZH2-induced proliferation, migration, and angiogenesis. Oncotarget. 2010; 1:710-720. https://doi.org/10.18632/ oncotarget.205.

18. Fan TY, Wang H, Xiang P, Liu YW, Li HZ, Lei BX, Yu M, Qi ST. Inhibition of EZH2 reverses chemotherapeutic drug TMZ chemosensitivity in glioblastoma. Int J Clin Exp Pathol. 2014; 7:6662-6670.

19. Hu P, Chu J, Wu Y, Sun L, Lv X, Zhu Y, Li J, Guo Q, Gong C, Liu B, Su S. NBAT1 suppresses breast cancer metastasis by regulating DKK1 via PRC2. Oncotarget. 2015; 6:3241032425. https://doi.org/10.18632/oncotarget.5609. 
20. Zang C, Nie FQ, Wang Q, Sun M, Li W, He J, Zhang M, $\mathrm{Lu}$ KH. Long non-coding RNA LINC01133 represses KLF2, P21 and E-cadherin transcription through binding with EZH2, LSD1 in non small cell lung cancer. Oncotarget. 2016; 7:11696-11707. https://doi.org/10.18632/ oncotarget. 7077 .

21. Shou J, Ali-Osman F, Multani AS, Pathak S, Fedi P, Srivenugopal KS. Human Dkk-1, a gene encoding a Wnt antagonist, responds to DNA damage and its overexpression sensitizes brain tumor cells to apoptosis following alkylation damage of DNA. Oncogene. 2002; 21:878-889.

22. Gomez-Manzano C, Lemoine MG, Hu M, He J, Mitlianga P, Liu TJ, Yung AW, Fueyo J, Groves MD. Adenovirallymediated transfer of E2F-1 potentiates chemosensitivity of human glioma cells to temozolomide and BCNU. Int $\mathrm{J}$ Oncol. 2001; 19:359-365.

23. Liu SM, Lu J, Lee HC, Chung FH, Ma N. miR-524-5p suppresses the growth of oncogenic BRAF melanoma by targeting BRAF and ERK2. Oncotarget. 2014; 5:9444-9459. https://doi.org/10.18632/oncotarget.2452.

24. Chen L, Zhang W, Yan W, Han L, Zhang K, Shi Z, Zhang J, Wang Y, Li Y, Yu S, Pu P, Jiang C, Jiang T, Kang C. The putative tumor suppressor miR-524-5p directly targets Jagged-1 and Hes-1 in glioma. Carcinogenesis. 2012; 33:2276-2282.
25. Cao R, Wang L, Wang H, Xia L, Erdjument-Bromage H, Tempst P, Jones RS, Zhang Y. Role of histone H3 lysine 27 methylation in Polycomb-group silencing. Science. 2002; 298:1039-1043.

26. Vire E, Brenner C, Deplus R, Blanchon L, Fraga M, Didelot C, Morey L, Van Eynde A, Bernard D, Vanderwinden JM, Bollen M, Esteller M, Di Croce L, et al. The Polycomb group protein EZH2 directly controls DNA methylation. Nature. 2006; 439:871-874.

27. Ning X, Shi Z, Liu X, Zhang A, Han L, Jiang K, Kang C, Zhang Q. DNMT1 and EZH2 mediated methylation silences the microRNA-200b/a/429 gene and promotes tumor progression. Cancer Lett. 2015; 359:198-205.

28. Lu J, Zhao FP, Peng Z, Zhang MW, Lin SX, Liang BJ, Zhang B, Liu X, Wang L, Li G, Tian WD, Peng Y, He ML, Li XP. EZH2 promotes angiogenesis through inhibition of miR-1/Endothelin-1 axis in nasopharyngeal carcinoma. Oncotarget. 2014; 5:11319-11332. https://doi.org/10.18632/ oncotarget. 2435.

29. Zhang X, Zhao X, Fiskus W, Lin J, Lwin T, Rao R, Zhang Y, Chan JC, Fu K, Marquez VE, Chen-Kiang S, Moscinski LC, Seto E, et al. Coordinated silencing of MYC-mediated miR-29 by HDAC3 and EZH2 as a therapeutic target of histone modification in aggressive B-Cell lymphomas. Cancer Cell. 2012; 22:506-523. 